\begin{tabular}{c} 
International Journal of Engineering \& Technology, 7 (2.7) (2018) 770-773 \\
International Journal of Engineering \& Technology \\
SPC \\
Website www.sciencepubco.com/index.php/IJET \\
Research Paper \\
\hline
\end{tabular}

\title{
Prevention of Spoofing Offensive in Wireless Sensor Networks
}

\author{
U.Lakshmi Sowmya ${ }^{1}$, M.Sai Kumar reddy ${ }^{2}$, K.Madhu Babu ${ }^{3}$, Dr.K.V.D.Kiran ${ }^{4}$ \\ Department of Computer Science and Engineering, \\ Koneru Lakshmaiah Education Foundation, \\ Guntur, Andhra Pradesh 522502, India \\ *Email ID : sowmyauppu7@gmail.com
}

\begin{abstract}
Remote sensor systems are powerless against assaults identified with data fraud and wholesale fraud. Albeit different techniques have been forced to recognize and find assailants, it doesn't concentrate on keeping the hub from assaulting. In this paper, we propose to demonstrate how the hostile of wholesale fraud and pantomime, for example, the surge hostile, happens when the surge hostile sends exhaust parcels to upset the information sent by the hub and side tracks the foe bundles at various way. Utilizing the neighboring hub signature confirmation strategy by mailing the keys to the hubs by key supplier whatever keeps the hub from being assaulted. The most limited way between the source hub and the goal hub was chosen utilizing the AODV. Counteractive action instrument will be exceptionally valuable for snappy distinguishing proof of assailants and furthermore enhance the execution of the structure.
\end{abstract}

Keywords: hubs; Sensor systems; signature; Techniques; AODV

\section{Introduction}

Remote sensor systems have as of late been engaged as a result of various zones accessible for the improvement of gadgets and in light of the difficulties in their outline. The hubs of the remote structure can be assaulted with the guide of cheap sensor gadgets in which the impersonation hostile can be effortlessly started and cause basic harm and impact the basic execution. The presumption of distinguishing the caricaturing hub must be intense, in light of the fact that some safety efforts are fundamental to keep away from these offensives, however the identification and area of the aggressors. are chiefly gone for keeping the hos- tile. the identification instrument is utilized when the hub is assaulted utilizing the RSS and bunch investigation technique to decide the quantity of aggressors and the incorporated strategy and recognition and limitation (IDOL) to discover the area of the assailants and requires numerous components for these procedures in these strategies we fathom the hostile that needs to do with impersonation and impersonation, for example, the hostile of the surge and the hostile hub hostile where the main character for pantomime was found in the past methodologies. The upsides of the present approach are that it can keep the hostile and thusly diminishes the additional expenses and modifications made by remote gadgets. The most essential commitment of our work is to exhibit the impersonation of personality and impersonation as the surge hostile. This surge hostile will attempt to keep the correct clients from accessing the wellsprings of the structure, after which the autonomous client won't react accurate- ly and will forward the information to another side. By utilizing the separation vector calculation to know the most limited separation and after-ward utilizing the mark confirmation strategy for the neighboring hub, you can keep away from the hostile.

\section{Existing System}

Nowadays, heterogeneous sensor frameworks can be found in machine settings, for instance, condition checking, cultivating, circulation focus following, transport collaborations, perception and restorative administrations. by and by we will inspect present account plans for spread sensor structure been remote the meaning of a scientific categorization of working programming for remote sensor information and an audit of a couple of basic subjects remote structure nearing accouterments structures inside the WSN, Internet-based compromise of sensor data in conclusion IP-based homogeneous mid-ware courses of action. Ensuing to scrutinizing this segment, the will know unequivocally what arrangements have been investigated and what particular guidelines and structures exist concerning middleware programming for remote and hybrid sensor frameworks.

\section{Wireless Sensor Networks}

Remote sensor mastermind (WSN) insinuates a social affair of spatially scattered and conferred sensors for watching and recording the physical conditions of nature and dealing with the assembled data at a central zone. WSNs measure regular conditions like temperature, sound, defilement levels, dampness, wind, and so on.These resemble exceptionally named remote frameworks as in they rely upon remote accessibility and unconstrained frameworks organization with the objective that 
sensor data can be transported remotely. Every so often they are insinuated as spotless nets and imply minor sensors as meager as clean. Sharp Dust [1] [2] [3] is a DARPA upheld U Berkeley wander. Clean Networks Inc. is one of the primary associations to make remote sensor things. WSNs are spatially passed on self-choice sensors to screen physical or normal conditions, for example, temperature, sound, weight, and whatnot., and to coordinate their information through the structure to a rule an area. The more present systems are bidirectional further more permit control of sensor advancement. The difference in remote sensor structures has been pushed by military contraptions, for example, the view of fights; Today, such systems are utilized as a bit of different mechanical and client gadgets, for example, introduce day process checking and control, machine viewing, and so on.

\section{Types of Offensive}

\subsection{Smurf Offensive}

The smurf threatening is a spread foreswearing of-advantage unfriendly in which broad amounts of Internet Control Message Protocol (ICMP) bundles with the delivered source IP of the proposed setback are transmitted to a PC structure using an IP convey ad-dress. Most gadgets on a structure will react as a matter of course by sending a reaction to the source IP address. In the event that the quantity of PCs in the structure accepting and reacting to these bundles is substantial, the casualty's PC will be over-burden with movement. This can back off the casualty's PC to the point where it is difficult to chip away.

\subsection{Arrange Cord Offensive}

Uncontrolled strategy string[1] is a kind of programming inadequacy found around 1989 that can be utilized as a bit of security mishandle. Starting at now thought safe, form string encounters can be utilized to crash a program or to execute dangerous code. The issue starts from the utilization of unchecked client duty as the strategy string parameter in certain $\mathrm{C}$ restrains that perform arranging, for example, printf(). A pernicious client may utilize the $\%$ s and $\%$ x plot tokens, among others, to print information from the call stack or possibly novel districts in memory. One may in like way frame subjective information to self-assured districts utilizing the \%n coordinate token, which charges printf() and comparable capacities to influence the sum to out of bytes dealt with to an address set away on the stack.

\subsection{DRWFLOODING OFFENSIVE:}

DRW Flood hostile are the most concerning issues in the field of security. These surges hostile make ex-press endeavors to irritate the right clients to get to the administrations. This hostile take control of structure hubs by abusing their vulnerabilities. A system as a rule requires a world- wide comprehension of the issue and methods to counteract hostile. There are two strategies to make the DRW hostile in the structure. The main strategy is that the aggressor sends void or irrelevant bundles to the hub to upset the convention that keeps running on it. It is likewise called the helplessness hostile. The second sort of unfriendly is the most generally perceived where the aggressor does the one they will do both in the going with courses: - By incapacitating the exchange speed and processor breaking point of the switch, we can aggravate the correct customers. By depleting server assets, for example, attachments, cpu, memory, the database will disturb the right clients and this will predominantly incorporate flooding hostile at the machine level. DRW over-burden hostile have been propelled in numerous associations. There are around 7000 DRW hostile watched day by day.

HUMAN OFFENSIVE IN THE MEDIUM Phishing is the way toward endeavoring to get delicate data, for example, secret key and user name by taking on the appearance of the trusted client so as to acquire a watchword.

Man-in-the-Middle offensive hostile is a champion among the most famous for phishing. Man-In-The-Middle undermining are the most well known and basic unpleasant on passed on getting ready movements. The Man- In-The Middle undermining is a threatening in which the interloper can read and shape messages when two social events chat with each other. The threatening shows up in different structures and structures because of the movement of figuring. For instance, in the http exchange, the TCP connection between the customer and the server is the objective. Utilizing the varying structures, the assailant breaks the main TCP relationship into two new affiliations. The first is between the customer and the assailant and the second is between the attacker and the server. By getting the TCP connection, the aggressor will go about as a center individual and will have the ability to scrutinize, create, and modify the data in the got correspondence. This threatening is to a great degree profitable in perspective of the possibility of http tradition and data trade which are inside and out in perspective of ASCII. By this, it is possible to see and to meet in the http tradition and moreover in the traded data

\section{Signature Verification}

Neighbor middlemost mark affirmation is the strategy which is done to keep the threatening instead of recognizing the unfriendly. By doing everything considered the exemplifying and the criticizing related hostile like the flooding adversarial and the man in the center debilitating are incredibly decreased .At first the center must be sent and from that point after the setting up the way where the information must be exchanged. The key supplier will give the keys to the midpoint focuses which was picked. The center point will influence the demand to the midpoint to point where the information must be exchanged by asking them the key and the middlemost point will be quickly react and a while later send the keys to the midpoint which ask the demand and after that inside which sends the deals will check the keys on the off chance that it attested then it will send the record or the information to the midpoint. In mechanized check the midpoint point A will encode the message by utilizing the private key of An and it appropriates general society key of A to different clients and they will unscramble the information with the comprehensive group key of A. Precisely when there is a known way and if there is no such satirizing adversarial the information exchange will oblige yet if there should be an occurrence of different information asking for to midpoint to get to the advantages by then there will be a befuddle to the mid point which sends the information, to which mid it need to send in light of the way that different middlemost are guaranteed to be a practically identical mid point. By then the sender who sends the information will sends a deals to every single one of the midpoint guides who expressed toward be an equivalent focus to send the check where essentially the primary focus have it. The middlemost focuses sends the key, the sender checks the keys send by the center and it locate the fundamental deepest and along these lines the joke center it send the information to the essential center and it contorts up discernibly effective and after that whatever is left of the center focuses are showed up as the sit out of gear center point to demonstrate that they are the aggressors. The deepest point which twists up detectably effective will begin asks the demand to the accompanying deepest like that the system proceeds until the 
point that it achieves the target methodology continues until the point that it accomplishes the objective

\section{Route Selection}

In remote sensors when two disjoint hubs speak with each other, the correspondence remove is restricted. hence, every hub in the structure needs to play as the host and the switch. Every hub needs to settle a course before sending the message. AODV is the steering system that passes messages to neighbors to hubs with which they can not impart straightforwardly. Ad hoc On-Demand Distance Vector (AODV) doing this by distinguishing the courses through which the message is passed. On Demand Vector might have the capacity to deal with changes in courses and make new courses if there is a blunder. The most productive conventions to get the briefest way and the least vitality utilization. It is fundamentally utilized as a part of impromptu systems and remote systems. The idea utilized as a part of this convention is the disclosure and upkeep of courses. You will discover courses just if there is a need. Utilize the grouping number to discover the precision of the data. It will take after the following bounce on the course as opposed to following the whole course. It will send occasional welcome messages to neighbors to refresh their hub position. To control the directing procedure, it doesn't require any focal managerial framework. It utilizes the steering table to store the directing data in the directing tables for the unicast courses and them directing tables for the multicast courses. In a few systems that are utilized to discover the course determination, for example, the separation vector on request, DSRs have been forced in which the AODV is forced through which the course can be productively chosen to exchange the information.

The coordinating table stores the objective locations, the accompanying bounce address, the objective progression number, Life Time. The life time fortified each time the course is utilized, if the course isn't utilized inside its lifetime, the course closes. In the event that there is an expansion in the movement number, it exhibits that there is an alteration in the topology. The AODV contains dominatingly two strategies, for example, course disclosure and course up- keep. Right when the center wishes to send groups to send a target, it checks its controlling table to pick whether it has a present course to the goal. In the event that yes, it impels the package to the

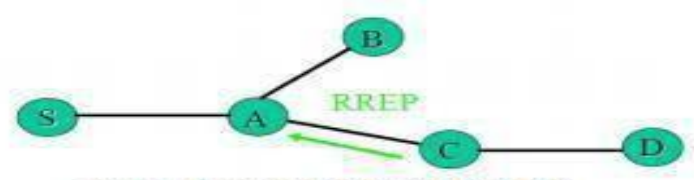

Fig 1.1 Sending the Route Request

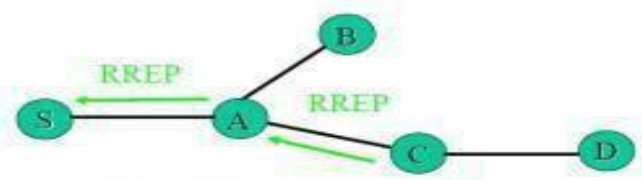

Fig 1.2 Forward Path Setup

going with skip center. If not, begin a course disclosure process. The course presentation process starts with the game plan of a course ask for separate that RREQ was made by the beginning focus. Once an inside point gets a RREQ, the center builds up a turn course region for the source center point in its course table. in the event that a $\mathrm{S}$ focus direct wishes toward send the information to middlemost point $\mathrm{D}$ sends the course demand to its neighboring focuses, course $\mathrm{C}$ gets the course demand and $\mathrm{C}$ makes the course reaction to focus point $\mathrm{A}$. A sends the course reaction to middlemost point $\mathrm{S}$ and midpoints gets the reaction from the course and plays out the section of the course of sending to course D, that is, it sends the bundle to target D. It diminishes the cost of developing inactivity in the look for new courses.

\section{Route Selection Algorithm}

Regardless of whether static, dynamic, or arrangement based directing is utilized, the calculation utilized by the IP layer to choose a course from a course table is the same. Course determination happens in the accompanying request:

On the off chance that a course exists to the goal address (a host course), it is picked.

On the off chance that no host course exists to the goal address, the course picked relies on the rendition of IP being utilized:

For IPv4: On the off chance that subnet, structure, or super network courses exist to the goal, the course with the most particular structure veil (the cover with the most bits on) is picked. On the off chance that the goal is a multicast goal and a multicast default course exists, that course is picked.

For IPv6, if prefix courses exist to the goal, the course with the most particular prefix is picked. Default courses are picked when no other course exists to a goal. Various equivalent cost courses are took into consideration static, dynamic, and strategy based directing. Different equivalent cost courses give extra data about the utilization of various equivalent cost courses. Without approach based

directing, the IP layer courses movement via hunting the fundamental course table down the most particular course known, utilizing the choice request depicted. On the off chance that strategy based steering is being utilized, the IP layer courses activity as per the arrangement characterized for the movement. For more data about how the IP layer courses movement when approach based steering is being utilized.

\section{Analysis and Implementation of WSN With Route Selection}

n a keen city framework, the Wireless Sensor Network (WSN) is the most vital system for gathering ecological information to give administrations. WSN is winding up progressively mainstream on account of its handiness and appeal. Since vitality assets are restricted in WSN, the outline of a WSN framework ought to think about both vitality proficiency and apparatus necessities. The adequacy of the framework depends completely on its gadget and equipment particulars; Therefore, WSN's directing calculation must be streamlined to accomplish vitality effective correspondence. The proposed Energy Use Oriented Route Selection (ECORS) calculation is the course choice calculation that endeavors to broaden framework life, particularly for information accumulation gadgets. This article proposes the proper information gathering instrument, with an accentuation on the usage, which incorporates the real bundle exchange component and the enhanced course determination work in an assumed situation. By changing the parameters with the possibility of execution, the proposed system can expand the lifetime by 2.03 times contrasted with the settled steering situation. What's more, the reenactment result is broke down in detail, concentrating on the charge minor departure from every hub. Also, the new pointer is exhibited, which speaks to the connection between the lifetime extension rate and 
the parameters utilized for the calculation. Utilizing this marker, the lifetime augmentation rate can be accepted without recreation, which is useful for the execution.

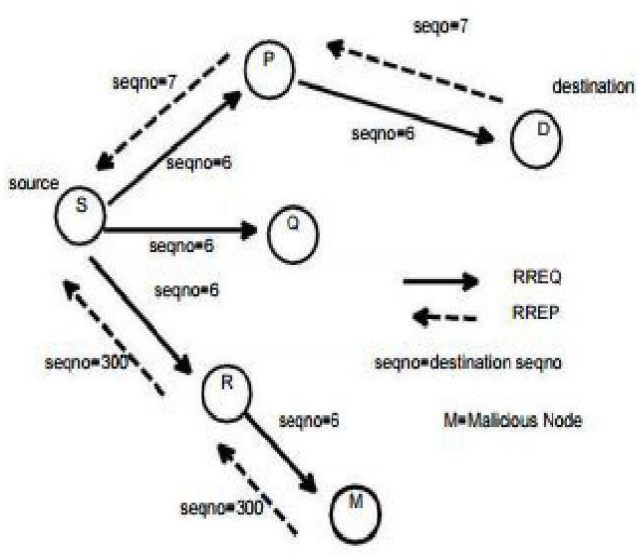

\section{Conclusion}

The hostile aversion system in wholesale fraud and the usurpation of assaults like surge and man in the center hostile are powerful contrasted with those of the discovery instrument where the hostile is recognized at that point deciding the quantity of aggressors and afterward the area of the assailant is acquired. By utilizing the neighboring hub signature confirmation technique, aggressors are distinguished and forestalled through which information is exchanged by means of the right hub. The level of execution of this counteractive action system is superior to anything that of the location instrument hypothetically got. It decreases time, cost and vitality utilization, and utilizing just this strategy for counteractive action cannot ensure that it can keep any hostile and there might be a remarkable possibility that the aggressor exists, so we can do this as future upgrade by utilizing the propelled location instrument whereby a few assailants existing in the anticipation component can be identified and avoid different hubs of the structure by giving cautioning data and fore- stalling them by giving an entrance to structure assets.

\section{Acknowledgement}

This work is supported by the Department of Science and Technology, India through the fund sanctioned for improvement of Science \& Technology infrastructure, at department of CSE, K.L University, by order number SR/FST/ESI-332/2013.

\section{References}

[1] Sharma.p,Trivedi,A "An Approach to Defend Against Warm hole offensive In Ad HoUsing The Digital Signature" Communication Software and Networks(ICCSN) 2011 IEEE 3rd International Conference on may 2011.

[2] Youngsoo Kim, Daejeon, Jungchan Na, Seungwon Sohn "A Secure Method For Transferring Active Packet Using The Digital Signature Schemes" Telecommunications, 2003. ICT 2003. 10th International Conference Vol.01 on 23 Feb 2003.
[3] Zhijun Li and Guang Gong "On The Node Clone Detection In Wireless Sensor Networks" IEEE/ACM Transactions on networking,

[4] Saman Taghavi Zargar, James Joshi, Da- vid Tipper "A Survey of Defence Mechanisms Against Distributed Denial of Service(DDoS) Floodis" IEEE Communications Surveys \& Tutorials Vol.15 Fourth Quarter 2013

[5] Joshi, Y, Das, D. ; Saha, S."Mitigating Man in the Middle offensive Over Secure Socket Layer" on Internet Multimedia Services Architecture and appliancess (IMSAA), 2009 IEEE International Conference on Dec.2009.

[6] Guha,R.K.Furqan, Zeeshan ,Muhammad, Shahabuddin "Discovering Man in The Middle Attacks in Authentication protocols"Military Communications Conference, 2007. MILCOM 2007. IEEE on 31 oct 2007.

[7] Chakeres, I.D. Belding-Royer, E.M. "AODV Routing Protocol Design Implementation" Distributed Computing Systems Workshops, 2004. Proceedings. 24th International Conference on march 2004

[8] Royer, E.M. "An Implementation Study of the AODV Routing Protocol" Wireless Communications and Networking Conference, 2000. WCNC. 2000 IEEE Vol.3 Sep 2003"

[9] K.V.D.KIRAN,"Integrated Distributed Architecture to Integrate Wireless Sensor Networks (WSN) with Grid for Healthcare," International Journal of BioScience and BioTechnology", Vol.7, No.3 (2015), pp.243-250, ISSN: 2233-7849 IJBSBT

[10] K.V.D.KIRAN,"A Critical study of information security risk assessment using fuzzy and entropy methodologies,"InternationalJournalon Computersand Communications". 